\title{
Prevalência de dor osteomuscular em idosos atendidos em uma unidade básica de saúde de Petrolina- PE
}

Prevalence of musculoskeletal pain in elderly patients cared for in a basic health unit in Petrolina-PE

\author{
Camila Silva Torres" $\bullet$
}

Fisioterapeuta, Petrolina, Pernambuco, Brasil. Camila Silva Torres. E-mail: Drcamilafisioterapeutaa@outlook.com

\begin{abstract}
Resumo: Introdução: A população está envelhecendo ligeiramente, dado esse processo, há o surgimento de problemas como as doenças osteomusculares. Os objetivos foi verificar a prevalência da dor osteomuscular em idosos atendidos em uma unidade básica de saúde na zona urbana de Petrolina-PE. Material e métodos: A variável dor musculoesquelética foi avaliada por meio de um questionário estruturado baseado na versão geral em português do Questionário Nórdico de Sintomas Osteomusculares (QNSO) adaptado transculturalmente para o Brasil. O Processamento e análise de dados foram com auxílio do Statistical Package for Social Sciences (SPSS) versão 16, após inserção através de digitação dupla. Resultados e discussão: A prevalência de dor osteomuscular em idosos atendidos em uma unidade básica de saúde de Petrolina Pe foi elevada, e o local do corpo mais referido foi a região dos joelhos. Considerações finais: Precisa-se de mais estudos que demonstrem essa prevalência em idosos com amostras maiores e abrangendo mais localizações.
\end{abstract}

Palavras-chaves: dor osteomuscular, idoso, prevalência.

\begin{abstract}
Introduction: The population is aging slightly, given this process, there are problems such as musculoskeletal diseases. The objective was to verify the prevalence of musculoskeletal pain in the elderly assisted at a basic health unit in the urban area of Petrolina-PE. Material and methods: The musculoskeletal pain variable was assessed using a structured questionnaire based on the general Portuguese version of the Nordic Musculoskeletal Questionnaire (QNSO) crossculturally adapted for Brazil. Data processing and analysis were performed using the Statistical Package for Social Sciences (SPSS) version 16, after insertion through double typing. Results and discussion: The prevalence of musculoskeletal pain in the elderly cared for in a basic health unit in Petrolina Pe was high, and the most referred body site was the knee area. Final considerations: More studies are needed to demonstrate this prevalence in the elderly with larger samples and covering more locations.
\end{abstract}

Keywords: musculoskeletal pain, elderly, prevalence.

\section{Introdução}

Os idosos correspondem a $11 \%$ da população total do país e necessitam dispor de prioridade quanto aos meios sociais e econômicos (Berlezi et al., 2019), esse envelhecimento acarreta o surgimento de problemas crônicos e degenerativos, como as doenças osteomusculares que são doenças prevalentes e sintomáticas, que causam quadros dolorosos (Litvak, 1990), de acordo com a International Association for the Study of Pain (IASP) a dor é uma sensação ou vivência insatisfatória, associada a lesão tecidual real ou potencial (Merskey \& Bogduk, 1994).

Os quadros de dor na doença osteomuscular, interferem na autonomia do idoso nas suas atividades de vida diária, onde causa a diminuição da qualidade de vida e prejudica a sua funcionalidade (De Carlo, 2004).

Tendo em vista a funcionalidade a Portaria Ministerial $\mathrm{n}^{\mathrm{o}} 1.271$, de 6 de junho de 2014, notificou 80,1\% de acometimento nesse aspecto relacionado a doenças osteomusculares em Pernambuco (Secretária Estadual de Saúde, 2016). Desse modo, ressalta-se a importância de conhecer a prevalência dessas condições na população para traçar medidas de tratamento e preventivas na atenção básica.

Algumas medidas podem incluir a atuação da fisioterapia em abordagens coletivas (Hasselkus, 1998) e atividades individuais com intuito de diminuir ou corrigir posturas deficitárias nas atividades de vida diária e mudanças comportamentais (Norburn et al., 1995), além de redução dos quadros álgicos, redução do cansaço e prevenção de patologias osteomusculares (Leal et al., 2018).

O presente estudo teve como objetivo verificar a prevalência da dor osteomuscular em idosos atendidos em uma unidade básica de saúde na zona urbana de Petrolina-PE. 


\section{Material e métodos}

\section{Delineamento do estudo}

Trata-se de um estudo de caráter descritivo com delineamento transversal.

\section{Local do estudo}

Unidade básica de saúde Dr. Manoel Possídio, rua Avenida dos Estados Unidos, no bairro: Areia Branca, na zona urbana do Município de Petrolina, Pernambuco.

\section{Período do estudo}

Foi realizado a Coleta de dados em outubro e novembro de 2018.

\section{População do estudo}

A população do estudo foi constituída por idosos com 60 anos ou mais, que residiam na zona urbana do Município de Petrolina, Pernambuco.

\section{Critérios de Elegibilidade Critérios de inclusão}

Foram adotados os seguintes critérios de inclusão: ser idoso (com idade igual a 60 anos ou mais); residir na zona urbana do município de Petrolina; ser funcionalmente ativo; apresentar função cognitiva satisfatória. A atividade funcional adequada foi definida como a capacidade de caminhar dentro e fora de casa sem dificuldade ou ajuda externa, e função cognitiva satisfatória foi definida como auto capacidade para interagir com o entrevistador (Almeida-Santos et al., 2016).

\section{Critérios de exclusão}

Foram excluídas do estudo os idosos que apresentaram restrições funcionais, déficits cognitivos e que se negaram a responder o questionário.

\section{Definição e operacionalização das variáveis Variável dependente}

Foi considerado variável dependente: a presença de dor osteomuscular.

\section{Variável independente}

Foi considerado variável independente: as variáveis sociodemográficas, o estado geral de saúde e o uso de medicamentos.

\section{Coleta de dados Instrumentos para coleta de dados}

A variável dor musculoesquelética foi avaliada por meio de um questionário estruturado baseado na versão geral em português do Questionário Nórdico de Sintomas Osteomusculares (QNSO) adaptado transculturalmente para o Brasil por Pinheiro et al. (2002).

\section{Avaliação sociodemográfica e do estado geral de saúde}

Para avaliação sociodemografica foi utilizado um questionário estruturado baseado nos critérios do Instituto Brasileiro de Geografia e Estatística (IBGE) (APÊNDICE B).

E para avaliação do Estado geral de saúde foi utilizado o questionário estruturado, contendo questões acerca da percepção de saúde, uso de medicamentos e presença de morbidades (APÊNDICE C).

Prevalência de dor osteomuscular em idosos atendidos em uma unidade básica

\section{Procedimentos}

Para apresentação e familiarização com o projeto, ocorreu inicialmente a divulgação em uma Unidade Básica de Saúde da zona urbana de Petrolina- PE.

Após a divulgação, foi feita a entrega da carta explicativa e do termo de consentimento livre e esclarecido para os idosos que se interessaram em participar do estudo. Em seguida, foi agendada a data para a avaliação que ocorreu em uma das salas de uma Unidade Básica de Saúde da Zona Urbana do município de Petrolina-PE. Os voluntários passaram por uma entrevista após a adesão à pesquisa, essa entrevista foi conduzida por um universitário para preenchimento de um questionário estruturado contendo questões referentes a dor músculo esquelética. 


\section{Processamento e análise dos dados}

Os dados foram analisados com auxílio do Statistical Package for Social Sciences (SPSS) versão 16, após inserção através de digitação dupla. Inicialmente, foi verificada a normalidade dos dados contínuos (ShapiroWilk). A análise descritiva para as variáveis categóricas incluiu a distribuição das frequências absolutas e relativas, e para as variáveis numéricas foram calculados os valores de tendência central e dispersão de acordo com a distribuição dos dados. Os intervalos de confiança de 95\% (IC 95\%) também foram calculados.

\section{Aspectos éticos}

Esta pesquisa foi aprovada pelo Comitê de Ética em Pesquisa da Faculdade de Ciências de Pernambuco (CEPFCM/ PE) sob o CAAE: 88147918.9.000.5192. Acrescido a isso, a pesquisa obedeceu a todos os preceitos da Resolução 466/12 do Conselho Nacional de Saúde (CNS). Os participantes assinaram termo de consentimento livre e esclarecido (TCLE). Para os participantes analfabetos que se interessaram em participar da pesquisa, foi realizada a leitura do termo de consentimento livre e esclarecido (TCLE) na presença de uma testemunha eleita pelo participante.

\section{Resultados e discussão}

Foram avaliados 40 idosos, 2 foram excluídos pois não aguardaram a conclusão da entrevista, sendo incluídos na análise final 38 indivíduos. A distribuição das características sociodemográficas e econômicas dos idoso avaliados estão apresentadas na Tabela 1.

Tabela 1. Distribuição das características sociodemográficas e econômicas dos idosos atendidos em uma UBS da zona urbana do município de Petrolina-PE ( $\mathrm{n}=38)$.

\begin{tabular}{|c|c|c|c|}
\hline & $\mathbf{n}$ & $\%$ & IC 95\% \\
\hline \multicolumn{4}{|l|}{ Sexo } \\
\hline Feminino & 28 & 73,7 & $57,99-85,03$ \\
\hline Masculino & 10 & 26,3 & $14,97-42,01$ \\
\hline \multicolumn{4}{|l|}{ Ocupação } \\
\hline Aposentado & 30 & 78,9 & $63,65-88,93$ \\
\hline Trabalha & 3 & 7,9 & $2,72-20,80$ \\
\hline Outra & 5 & 13,2 & $5,75-27,33$ \\
\hline \multicolumn{4}{|l|}{ Religião } \\
\hline Católica & 34 & 89,5 & $75,87-95,83$ \\
\hline Evangélica & 4 & 10,5 & $4,17-24,13$ \\
\hline \multicolumn{4}{|l|}{ Estado conjugal } \\
\hline Solteiro & 6 & 15,8 & $7,44-30,42$ \\
\hline Casado ou União estável & 17 & 44,7 & $30,15-60,29$ \\
\hline Viúvo & 9 & 23,7 & $12,99-39,21$ \\
\hline Outro & 6 & 15,8 & $7,44-43,71$ \\
\hline \multicolumn{4}{|l|}{ Escolaridade } \\
\hline Analfabeto ou Fundamental incompleto & 16 & 41,2 & $27-85-57,81$ \\
\hline Fundamental completo ou Médio incompleto & 8 & 21,0 & $11,07-36,35$ \\
\hline Médio completo ou Superior incompleto & 8 & 21,0 & $11,07-36,35$ \\
\hline Superior Completo & 6 & 15,8 & $7,44-43,71$ \\
\hline \multicolumn{4}{|l|}{ Renda familiar mensal em salários mínimos (SM*) } \\
\hline Menos de 1SM & 5 & 13,2 & $5,75-27,33$ \\
\hline 1 a 2 SM & 27 & 71,0 & $55,24-83,00$ \\
\hline$>2 \mathrm{SM}$ & 6 & 15,8 & $7,44-43,71$ \\
\hline
\end{tabular}

*SM, salário mínimo baseado no valor de R\$954,00 (Coleta realizada em 2018).

A mediana da idade foi 68, 5 anos (Valor mínimo 60,0 - Valor máximo 92,0). Em relação as variáveis antropométricas, a mediana da massa corporal foi 68,2 kg (Valor mínimo 40,8 - Valor máximo 94,0) e da estatura 1,55 m (Valor mínimo 1,42 - Valor máximo 1,85). A Tabela 2 apresenta a distribuição das características relacionadas ao estado geral de saúde e uso de medicamentos dos idosos avaliados.

Tabela 2. Distribuição das características relacionadas ao estado geral de saúde e uso de medicamentos e dor em idosos do município de Petrolina-PE $(\mathrm{n}=38)$.

$\begin{array}{llll}\text { Variáveis } & \mathrm{N} & \% & \text { (IC 95\%) }\end{array}$




\begin{tabular}{crrr}
\hline Como considera a saúde & & & \\
Muito boa ou boa & 12 & 31,6 & $19,08-47,46$ \\
$\quad$ Regular & 22 & 57,9 & $42,19-72,15$ \\
Ruim ou muito ruim & 4 & 10,5 & $4,17-24,13$ \\
Comorbidades & & & \\
Hipertensão & 23 & 60,5 & $44,72-74,40$ \\
$\quad$ Diabetes & 14 & 36,8 & $23,38-52,72$ \\
Uso de Medicamentos & & & \\
Sim & 32 & 84,2 & $69,58-92,56$ \\
Não & 6 & 15,8 & $7,44-30,42$ \\
Sim & & & \\
Não & 29 & 78,4 & $62,80-88,61$ \\
Dor nos últimos 12 meses & 8 & 21,6 & $11,39-37,20$ \\
Sim & & & \\
Não & 16 & 43,2 & $28,67-59,09$ \\
Dor nos últimos 7ias & 21 & 56,8 & $40,91-71,33$ \\
\hline
\end{tabular}

Dos avaliados 78,4\% (IC95\% 62,80 - 88,61) relataram dor nos últimos 12 meses, destes 61,1\% (IC95\% 44,86 - 75,22) procuraram um profissional de saúde para tratamento dos sintomas, e 24,3\% (IC95\% 13,36 - 40,12) referiram dor em dois ou mais segmentos do corpo. Em relação ao local da dor 13, 5\% (IC95\% $5,91-27,89)$ referiram dor nos joelhos, 10 , 8\% (IC95\% 4,29 - 24,71) referiram dor na coluna lombar e 10 , $8 \%$ (IC95\% 4,29 - 24,71) dor na coluna punho ou mão, esses foram os seguimentos mais citados.

Dos idosos que relataram dor nos últimos 12 meses 16,2\% (IC95\% 7,65 - 31,14) deixaram de trabalhar/ realizar alguma atividade devido a dor. Entre os indivíduos que referiram dor nos sete dias anteriores a entrevista 16,2\% (IC95\% 7,65 - 31,14) referiram dor em dois ou mais segmentos do corpo.

A auto percepção de saúde dos idosos está ligada a aspectos sociodemográficos, econômicos, psicológicos e de capacidade física (Jerez-Roig et al., 2016). Existem fortes evidências que esteja relacionada ao número de doenças crônicas e incapacidade funcional que pode ter como consequência quadros álgicos (Pagotto et al., 2011).

A dor presente na vida do idoso, limita sua qualidade de vida, restringe a sua independência e autonomia (Celich \& Galon, 2009). Dos idosos avaliados 78,4\% relataram dor nos últimos doze meses, destes $61,1 \%$ procuram profissionais de saúde, verifica-se que há uma interação dos mesmos com os profissionais de saúde. Isso é importante pois segundo Araújo e Barbosa (2010), o envolvimento entre o profissional e o paciente, influência a mudança de comportamento possibilitando o idoso sentir -se digno da sua autonomia e melhora a sua qualidade de vida. Há uma possibilidade de construção de respostas assistenciais e otimização do atendimento e do cuidado (Motta et al., 2008).

Em relação a dor, dentre os idosos avaliados 13,5\% informaram ter dor nos joelhos. Em geral a dor nos joelhos em idosos é associado ao desequilíbrio muscular proveniente do processo de envelhecimento natural (Slemenda et al., 1997). Nos casos mais álgicos pode ser considerado o uso de muletas para proteção da articulação, tendo em vista que o tratamento fisioterapêutico tem como objetivo: melhorar a postura e a musculatura destes pacientes, adequando o joelho a insuficiência estrutural da idade (Camanho, 2008).

Outros estudos relatam prevalência de dores nos joelhos em idosos variando entre 12 e $48 \%$ (Anderson et al.,1993; Woo et al., 1994). Outras partes do corpo foram verificadas no presente estudo com maiores prevalências álgicas, como a coluna lombar, punho ou mão.

Predominantemente no idoso a dor na coluna lombar é causada pela degeneração das estruturas da coluna vertebral que causa achatamento dos corpos vertebrais, perda de massa óssea, alterações discais e ligamentares, podendo ficar propenso a fraturas (Castro, 2000). E a eficiência biomecânica do disco intervertebral é diminuída com o decorrer do tempo devido ao aumento do estresse mecânico e a desidratação, comprometendo sua integridade e predispondo a hérnia (Cecin, 1997).

Diversos são os tratamentos empregados, desde a ingestão de medicamentos à terapêuticas de reabilitação englobando exercícios em vários segmentos, os exercícios diminuem a intensidade da dor lombar e auxiliam na recuperação do paciente, tais tratamentos incluem: laser de baixa potência, estimulação elétrica, termoterapia, educação postural e exercícios de fortalecimento geral (Kuijpers et al., 2011).

Em estudos feitos com adultos, em que se incluíram idosos, a prevalência de dor lombar variou de 14 a 49\% (Von Korff et al., 1988; Anderson et al.,1993; Badley \& Tennant, 1992). 
Em pesquisa realizada por Alves et al. (2009), observou-se as seguintes porcentagens de prevalência de dor osteomuscular em idosos: coluna lombar (30,43\%), ombros (25\%), punhos/mãos e coluna torácica (20\%).

O presente estudo obteve como fator de limitação o pequeno número amostral de idosos. Sugere-se que estudos futuros que avaliem essa prevalência em idosos com o número amostral maior e englobe mais localizações.

\section{Considerações finais}

O estudo apresentou uma elevada prevalência de dor osteomuscular entre os idosos avaliados, e o local do corpo mais referido foi a região dos joelhos, sugere-se um programa de assistência fisioterapêutica e multidisciplinar com enfoque nas áreas mais afetadas como medida de tratamento e de prevenção.

\section{Referências}

Almeida-Santos, M. A., Barreto-Filho, J. A., Oliveira, J. L., Reis, F. P., Cunha Oliveira, C. C., \& Sousa, A. C. 2016. Aging, heart rate variability and patterns of autonomic regulation of the heart. Archives of Gerontology and Geriatrics, 63, 1-8.

Alves, M. I. 2009. Compreender a Dor Neuropática Crónica Revisão bibliográfica.

Andersson, H. I., Ejlertsson, G., Leden, I., \& Rosenberg, C. 1993. Chronic pain in a geographically defined general population: studies of differences in age, gender, social class, and pain localization. The Clinical Journal of Pain, 9(3), 174-82.

Araújo, M. A. S., \& Barbosa, M. A. 2010. Relação entre o profissional de saúde da família e o idoso. Escola Anna Nery, 14(4), 819-824.

Badley, E. M., \& Tennant, A. 1992. Changing profile of joint disorders with age: findings from a postal survey of the population of Calderdale, West Yorkshire, United Kingdom. Annals of the Rheumatic Diseases, 51(3), 366-371.

Berlezi, E. M., Gross, C. B., Pimentel, J. J., Pagno, A. R., Fortes, C. K., \& Pillatt, A. P. 2019. Estudo do fenótipo de fragilidade em idosos residentes na comunidade. Ciência \& Saúde Coletiva, 24, 4201-4210.

Camanho, G. L. 2008. Dor aguda no joelho do paciente idoso. Revista Brasileira de Ortopedia, 43(9), 361-366.

Castro, M. 2000. A coluna lombar do idoso. Revista Brasileira de Ortopedia, 35(11/12), 423-425.

Cecin, H. 1997. Proposição de uma reserva anatomofuncional, no canal raquidiano, como fator interferente na fisiopatologia das lombalgias e lombociatalgias mecânicodegenerativas. Revista da Associação Médica Brasileira, 43(4), 295-310.

Celich, K. L. S., \& Galon, C. 2009. Chronic pain in elderly and its influence in daily activities and social living. Revista Brasileira de Geriatria e Gerontologia, 345-359.

De Carlo, M. 2004. O tratamento não farmacológico da dor e a abordagem da terapia ocupacional. São Paulo.

Hasselkus, B. 1998. Discapacidad funcional en ancianos. In: Hopkins, H. L., \& Smith, H.D. Terapia ocupacional de wilard e Spackman. Madrid: Panamericana, $8^{\mathrm{a}}$ ed.

Jerez-Roig, J., Souza, D. L., Andrade, F. L., Lima Filho, B. F., Medeiros, R. J., Oliveira, N. P., Cabral Neto, S. M., \& Lima, K. C. 2016. Self-perceived health in institutionalized elderly. Ciência \& Saúde Coletiva, 21(11), 3367-3375.

Kuijpers, T., van Middelkoop, M., Rubinstein, S. M., Ostelo, R., Verhagen, A., Koes, B. W., \& van Tulder, M. W. 2011. A systematic review on the effectiveness of pharmacological interventions for chronic nonspecific low-back pain. European spine journal: official publication of the European Spine Society, the European Spinal Deformity Society, and the European Section of the Cervical Spine Research Society, 20(1), 4050.

Leal, B., Guerin, I., Figueiredo, N., Souza, T., \& Pires, A. 2018. Atuação da fisioterapia preventiva em ler/dort no âmbito empresarial. URI São Luiz Gonzaga. Disponível em: < http://urisaoluiz.com.br/site/atuacao-dafisioterapia-preventiva-em-lerdort-no-ambito-empresarial/>. Acesso em:

Litvak, J. 1990. El envejecimiento de la población: um desafio que va más allá del ano 2000. Boletín de la Oficina Sanitaria Panamericana, 109(1), 1-5. 
Merskey, H., \& Bogduk, N. 1994. Classification of chronic pain: descriptions of chronic pain syndromes and definitions of pain terms. Seattle: IASP press.

Motta, L. B. D., Caldas, C. P., \& Assis, M. D. 2008. A formação de profissionais para a atenção integral à saúde do idoso: a experiência interdisciplinar do NAI-UNATI/UERJ. Ciência \& Saúde Coletiva, 13, 1143-1151.

Norburn, J. E., Bernard, S. L., Konrad, T. R., Woomert, A., DeFriese, G. H., Kalsbeek, W. D., Koch, G. G., \& Ory, M. G. 1995. Self-care and assistance from others in coping with functional status limitations among a national sample of older adults. Journals of Gerontology, Series B, Psychological Sciences and Social Sciences, 50(2), S101-109

Pagotto, V., Nakatani, A. Y. K., \& Silveira, E. A. 2011. Factors associated with poor self-rated health in elderly users of the Brazilian Unified National Health System. Cadernos de Saúde Pública, 27(8), 1593-1602.

Pinheiro, F. A., Tróccoli, B. T., \& Carvalho, C. V. 2002. Validação do Questionário Nórdico de Sintomas Osteomusculares como medida de morbidade. Revista de Saúde Pública, 36(3), 307-312.

Secretaria Estadual de Saúde. 2016. Perfil Socioeconômico, Demográfico e Epidemiológico: Pernambuco (1a ed.). Recife, PE: Secretaria de Saúde do Estado de Pernambuco.

Slemenda, C., Brandt, K. D., Heilman, D. K., Mazzuca, S., Braunstein, E. M., Katz, B. P., \& Wolinsky, F. D. 1997. Quadriceps weakness and osteoarthritis of the knee. Annals of Internal Medicine, 127(2), 97-104.

Von Korff, M., Dworkin, S. F., Le Resche, L., \& Kruger, A. 1988. An epidemiologic comparison of pain complaints. Pain, 32(3), 173-83.

Woo, J., Ho, S. C., Lau, J., \& Leung, P. C. 1994. Musculoskeletal complaints and associated consequences in elderly chinese aged 70 years and over. Journal of Rheumatology, 21(10), 1927-1931.

\section{Minicurrículo}

Camila Silva Torres. Formação do ensino médio - 2012 na Instituição Adventista Nordeste Brasileira de Educação e Assistência Social, graduação em Bacharelado em Fisioterapia - 03-2021 na Faculdade UniBRAS da Bahia (UniBRAS), pós-graduanda em Fisioterapia na Saúde da Mulher na Faculdade Venda Nova do Imigrante (FAVENI), pós-graduanda em Fisioterapia em Pediatria e Neonatal pela Faculdade Atualiza cursos. Possui Registro de conselho profissional- Crefito-1 332822-F e registro de pesquisadora- Orcid.org/00000002-1048-7145, realizou monitoria na disciplina de Eletrotermofototerapia em 2018 e 2019 e monitoria na disciplina de Cinesioterapia I e II em 2019, conta com grandes habilidades de comunicação, além de uma abordagem focada e estratégica em ambientes hospitalares, clínicas e unidade básica de saúde. Especializada em uma ampla gama de terapias e exercícios, e capaz de manter a motivação e a adesão dos pacientes ao longo dos planos de tratamento, apto a monitorar resultados para a manutenção da saúde e bem-estar, capaz de trabalhar em equipe e acompanhar cada paciente.

Como citar: Torres, C.S. 2022. Prevalência de dor osteomuscular em idosos atendidos em uma unidade básica de saúde de Petrolina- PE. Pubsaúde, 8, a250. DOI: https://dx.doi.org/10.31533/pubsaude8.a250

Recebido: 08 set. 2021.

Revisado e aceito: 26 set. 2021.

Conflito de interesse: os autores declaram, em relação aos produtos e companhias descritos nesse artigo, não ter interesses associativos, comerciais, de propriedade ou financeiros que representem conflito de interesse.

Licenciamento: Este artigo é publicado na modalidade Acesso Aberto sob a licença Creative Commons Atribuição 4.0 (CC-BY 4.0). 